\title{
EQUALITY IN BETWEEN ITERATIVE SOFT EROSION, ITERATIVE SOFT OPEN IN MULTI SCALE ENVIRONMENT
}

\author{
Kompella Venkata Ramana ${ }^{1}$

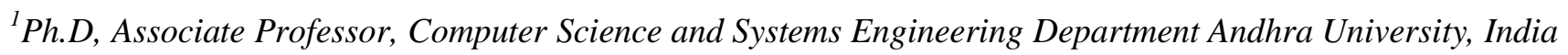

\begin{abstract}
This paper will come under discussion of fundamental properties of a narrow area in image processing. In this paper, equality is established and discussed in between soft erosion and soft open in multi scale environment as well as in iterative environment. Soft erosion and soft dilation will exist for various thresholds. So soft open also exist for various thresholds. If definition for soft erosion and soft dilation are studied (5), then some type of equalities are viewed among soft morphological operations. So equality may be established in between soft erosion and soft dilation in multi scale environment (47). In the same way because soft open is a composite operation, equality may be established among them also. In this paper it is extended to iterative environment. A very important point is that equality does not exist in mathematical morphology but will exist in soft mathematical morphology.
\end{abstract}

Keywords: Mathematical Morphology, Mathematical Soft Morphology, Soft Morphology, Erosion, Dilation, Soft Erosion, Soft Dilation, Soft Open, Primitive Morphological Operation, Equality, Threshold, Iterative Morphology,

Multi Scale Morphology.

\section{INTRODUCTION AND MATHEMATICAL MORPHOLOGY}

The human beings have the desire of recording incidents, through images. It has started from early cavemen also. Later, so many techniques, to get the images and so many techniques, to process the images are developed. After assembling of computers, image processing was expanded.

In 1964 G. Matheron was asked to investigate the relationships between the geometry of porous media and their permeability. At the same time, J. Serra was asked to quantify the petrography of iron ores, in order to predict their milling properties. $(1,2)$ At the same time a centre was developed to study mathematical morphological techniques in Paris school of mines, France. Mathematical morphology can provide solutions to many tasks, where image processing can be applied, such as in remote sensing, optical character recognition, Radar image sequence recognition, medical image processing etc.,

In mathematical morphological operations, Erosion and Dilation are primitive operations $(3,4)$. Open and close are composite operations.

Dilation: - These operations may be defined in so many ways. Different researchers defined this operation in different ways.

Def. 1:- Let $A$ and $B$ be subjects of $E^{N}$ (where $N$ is Space) the dilation of $\mathrm{A}$ by $\mathrm{B}$, is denoted by $\mathrm{A} \oplus \mathrm{B}$ and is defined by $\mathrm{A} \oplus \mathrm{B}=\{\mathrm{C} / \mathrm{C}=\mathrm{a}+\mathrm{b}$ for some $\mathrm{a} \in \mathrm{A}$ and $\mathrm{b} \in \mathrm{B}\}$

Def 2:- $A \oplus B=U(A)_{b}$ $\mathrm{b} \in \mathrm{B}$ Where $\mathrm{A}$ is the image and $\mathrm{B}$ is the structuring element.

Here $(A)_{b}$ means, translation of $A$ by $b$, defined as

$(\mathrm{A})_{\mathrm{b}}=\{\mathrm{C} / \mathrm{C}=\mathrm{a}+\mathrm{b} ; \mathrm{a} \in \mathrm{A}\}$

Def 3:- $\quad(I \oplus \mathbf{S})[x, y]=1$ if $\left|I \cap \mathbf{S}_{(x, y)}^{\prime}\right| \geq \mid$

$=0$ otherwise.

Here, $\mathrm{I}$ is the image

$S$ : structuring element

$\mathbf{S}^{\prime}$ : reflection of $\mathrm{S}$ about the origin

[If S.E. is having origin, at its centre point then $\mathbf{S}=\mathbf{S}^{\prime}$.]

$\mathrm{I}(\mathrm{x}, \mathrm{y})$ denotes image pixel value at the coordinate $(\mathrm{x}, \mathrm{y})$

$|Z|$ denotes the cardinality of the set $Z$;

$\mathrm{S}_{(\mathrm{x}, \mathrm{y})}: \mathrm{S}$ translated by the displacement $\{\mathrm{x}, \mathrm{y}\}$.

Erosion: This morphological operation also defined in so many ways, by different researchers.

Def 1):- The erosion of $A$ by $B$ is denoted by $A \ominus B$, and is defined by

$\mathrm{A} \ominus \mathrm{B}=\{\mathrm{x} / \mathrm{x}+\mathrm{b} \in \mathrm{A}$ for every $\mathrm{b} \in \mathrm{B}\}$ Here $\mathrm{x} \in \mathrm{E}^{\mathrm{N}}$ when $\mathrm{E}^{\mathrm{N}}=$ $\mathrm{N}$ space.

Def 2): $A \ominus B=\{x /$ for every $b \in B$, there exists and $a \in A$, such that $\mathrm{x}=\mathrm{a}-\mathrm{b}\}$

Def 3):- $(I \ominus S)[x, y]=1 \quad$ If $\left|I \cap S_{(x, y)}\right|=|S|$

$=0$ other wise

Here $\mathrm{I}$ is image and $\mathrm{S}$ is S.E. $\quad \mathrm{I}(\mathrm{x}, \mathrm{y})$ denotes image value at coordinate $(\mathrm{x}, \mathrm{y})$

|Z| denotes the cardinality, of the set $\mathrm{Z}$.

$\mathrm{S}_{(\mathrm{x}, \mathrm{y})}$ : $\mathrm{S}$ translated by the displacement $(\mathrm{x}, \mathrm{y})$

OPEN: It is a composite morphological operation. Open can be defined by the two primitive morphological operations, dilation and erosion operations. 
Def: Open can be defined as, Erosion on the image, followed by Dilation. Open can be represented as, "O" symbol. B O K means image " $\mathrm{B}$ " is opened by the structuring element " $\mathrm{K}$ "

\section{$\mathrm{B}$ O $\mathrm{K}=(\mathrm{B} \ominus \mathrm{K}) \oplus \mathrm{K}$}

\section{CLOSE}

It is also a composite morphological operation.

Def:- "Close" can be defined as, dilation on the image, followed by, erosion.

Close can be represented as $\quad$ symbol.

$\mathrm{B} \bullet \mathrm{K}$ means, image "B" is closed by the S.E., "K".B $\mathrm{K}=(\mathrm{B} \oplus \mathrm{K}) \ominus \mathrm{K}$.

\section{SOFT MORPHOLOGY}

In mathematical morphology, some type of the concept "All" will play major role.In Erosion, the O.P. will be "1", if all elements of the sub image are equal to 1 , otherwise, the output will be " 0 ". In dilation, the O.P. will be " 0 ", if all elements of the sub image are equal to " 0 ". Otherwise the output will be "1". This "All" concept, will cause some type of inconvenience. So some type of flexibility is introduced, in the form of threshold value. So, this morphology with threshold is defined as soft morphology. So, this soft morphology is having a few advantages, which the mathematical morphology operations don't have.

So, the Soft Morphology can be considered as extension to mathematical morphology. Even though mathematical morphological operators are efficient, they suffer with a few drawbacks as specified above. In addition to above, some more comments are. In primitive morphological operations, erosion, one or two mismatched pixels of image prevent the structuring element from fitting perfectly. It is the basic morphological operation, quantifies the way in which, the structuring element fits into the image. Erosion is an "All or nothing" transformation, implemented using bitwise "and". So, erosion will be sensitive to noise.

In primitive morphological operations, dilation, isolated pixels, even though, they are irrelevant to the image's content, significantly affect the output of the transformation. The net effect is an increased number of large spurious particles, increasing the confusion in the dilated image. So, noise will be added, which may be named as additive noise. (5).

But, many applications require more tolerance to noise than is provided by erosion and dilation. Soft morphological operators possess many of the characteristics, which are desirable, perform better in noisy environments. (5)

So, the soft morphological filters, improve the behavior of standard morphological filters, in noisy environment. The soft morphological filters are better compared to mathematical morphology in small detail preservation and impulse noise. In soft morphology, it preserves details, by adjusting its parameters (11). It can be designed in such a way that, it performs well in removal of salt - and - pepper noise as well as Gaussian noise, simultaneously. (12)
The idea of soft morphological operations is to relax, the standard morphological definition, a little, in such a way that, a degree of Robustness is achieved, While, most of the desirable properties of standard morphological operations are maintained. The soft morphology was introduced by KOSKINEN etc, and developed by researchers.

\subsection{Discussion on Defintion}

In some papers, researchers proposed soft morphology using two sets of structuring elements.

A) The core

B) The soft boundary $[7,8,9$ etc $]$.

But, in some papers [5 etc.] they proposed soft morphology, by counting logic. They have done the counting of ones, in the particular sub image, chosen. Then they have applied threshold value, for soft Erosion and soft dilation.

Soft dilation was defined as (5)

$\left(\mathrm{I} \bigoplus \mathrm{S}^{(\mathrm{m})}\right)[\mathrm{x}, \mathrm{y}]=1$ If $\left|\mathrm{I} \cap \mathrm{S}_{(\mathrm{x}, \mathrm{y})}\right| \geq \mathrm{m}$

$=0$ otherwise.

Here " $\mathrm{m}$ " is threshold value where $1 \leq \mathrm{m} \leq|\mathrm{S}| .|\mathrm{S}|$ is the cardinality of S.

Soft Erosion may be defined as

$\left(\mathrm{I} \ominus \mathrm{S}^{(\mathrm{m})}\right)[\mathrm{x}, \mathrm{y}]=0$ If $\left|\overline{\mathrm{I}} \cap \mathrm{S}_{(\mathrm{x}, \mathrm{y})}\right| \geq \mathrm{m}$

$=1$ otherwise. $\overline{\mathrm{I}}=$ inversion of $\mathrm{I} ; \mathrm{m}=$ threshold $\leq|\mathrm{S}|$.

[The exact definition, given in " 5 ", is slightly modified, according to the requirement, but without changing the meaning. Here the main assumption is origin is at central place of the structuring element and structuring element is assumed to be a square grid.]. Here " $m$ " is threshold value. Soft open and soft close are defined by above soft erosion and soft dilation...Some convention is discussed in the next section.

\section{ITERATIVE SOFT MORPHOLOGY}

It can be defined as, applying a morphological operation on an image, a few number of times.

\subsection{Convention}

Symbolically, $(\mathrm{X} \ominus \mathrm{Y})$ means applying erosion by S.E. Y, on image X.(XӨ2Y) means, applying Erosion by S.E. Y, on image $\mathrm{X}$, twice. (XӨ3Y) means, applying Erosion by S.E. $\mathrm{Y}$, on image $\mathrm{X}$, thrice. (XӨNY) means, applying Erosion by S.E. Y, on image "X", "N" number of times, in the same way.

$(\mathrm{X} \oplus \mathrm{NY})$ means, applying dilation by S.E. Y, on image "X" ,N no of times.(X O NY) means, applying open by S.E. $\mathrm{Y}$, on image " $\mathrm{X}$ ", $\mathrm{N}$ numbers if times. [But it is idempotent operation.] (X $\bullet \mathrm{NY}$ ) means applying close by S.E. Y, on image " $\mathrm{X}$ ", $\mathrm{N}$ number of times. [But it is also idempotent operation.] This iterative morphology will have applications in the design of composite morphological operations (Morphological Algorithms) skeletenization, thinning, thickening etc.

The applications may also be seen in structuring element Decomposition, segmentation, etc. 
Iterative morphology may be extended to iterative soft morphological environment also. In iterative soft morphological environment, the following convention may be used.

$\left(\mathrm{E}_{(1)}\right)^{2}$ : Soft Erosion, with threshold value $=1$ applied, 2 times on the image.

$\left(\mathrm{E}_{(1)}\right)^{5}$ : Soft Erosion, with threshold value $=1$ applied, 5 times on the image.

$\left(\mathrm{E}_{(\mathrm{x})}\right)^{\mathrm{y}}$ : Soft Erosion, with threshold value " $\mathrm{x}$ ", applied " $\mathrm{y}$ " times on the image.

$\mathrm{E}_{(1)}, \mathrm{E}_{(2)}, \mathrm{E}_{(3)}$, : Soft Erosion, applied with threshold values, $1,2,3$ on the image.

$\mathrm{E}_{(\mathrm{x})}, \mathrm{E}_{(\mathrm{y})}, \mathrm{E}_{(\mathrm{z})}$, : Soft Erosion, applied with threshold values, $\mathrm{x}, \mathrm{y}, \mathrm{z}$ on the image.

$\left(D_{(1)}\right)^{3}$ : Soft Dilation, with threshold value "1" applied " 3 " times on the image.

$\left(D_{(2)}\right)^{4}$ : Soft Dilation, with threshold value $=2$, applied, "4" times on the image.

$\left(E_{(x)}\right)^{y}$ : Soft Dilation, with threshold value $=x$, applied " $y$ " times on the image.

$\mathrm{D}_{(1)}, \mathrm{D}_{(2)}, \mathrm{D}_{(3)}$ : Soft Dilation, applied with threshold values, $1,2,3$ on the image.

$\mathrm{D}_{(\mathrm{x})}, \mathrm{D}_{(\mathrm{y})}, \mathrm{D}_{(\mathrm{z}) \text { : }}$ Soft Dilation, applied with threshold values $\mathrm{x}$, $\mathrm{y}, \mathrm{z}$ on the image.

$(\mathrm{O}(1,2))^{3}$ : Soft open applied thrice on the image, with thresholds 1,2

[Soft Erosion threshold value $=1$, Soft Dilation threshold value $=2]$

$(\mathrm{O}(\mathrm{x}, \mathrm{y}))^{\mathrm{n}}$ : Soft open, applied ' $\mathrm{n}$ " times, on the image, with thresholds $\mathrm{x}, \mathrm{y}$

[Soft Erosion threshold value $=\mathrm{x}$, Soft Dilation threshold value $=\mathrm{y}]$

$\mathrm{O}(\mathrm{p}, \mathrm{q}) \mathrm{O}(\mathrm{x}, \mathrm{y})$ : Soft Open applied twice on the image, with different thresholds.

$\mathrm{O}(\mathrm{p}, \mathrm{q}) \mathrm{O}(\mathrm{r}, \mathrm{s}) \mathrm{O}(\mathrm{x}, \mathrm{y})$ : Soft open, applied thrice on the image, with different thresholds.

$(C(1,2))^{4:}$ Soft close applied four number of times on the image, with Soft dilation threshold value $=1$, Soft Erosion threshold value $=2$.

$[C(1,2)]^{\text {n}}$ : Soft close applied " $n$ " number of times, on the image with thresholds 1, 2.

$(\mathrm{C}(\mathrm{x}, \mathrm{y}))^{\mathrm{n}}$ : Soft close applied " $\mathrm{n}$ " times, on the image, with thresholds $\mathrm{x}, \mathrm{y}$.

$\mathrm{C}(\mathrm{p}, \mathrm{q}) \mathrm{C}(\mathrm{r}, \mathrm{s}) \mathrm{C}(\mathrm{t}, \mathrm{u})$ : Soft close applied on the image, thrice, with different thresholds.

\subsection{Review On Iterative Soft Morphology}

A few researchers developed a process for convergence criteria, in iterative morphology. In iterative morphology, the system has to go towards a better solution. It is discussed in the paper (13). Another set of researchers have proposed an algorithm in iterative morphological environment, for segmentation.(15).

Another set of researchers (14), discussed methodology for segmentation technique, which is suitable to apply on sequence of images of traffic scenes. YONG XIA \& others (15), discussed methodology using multi fractal estimation technique for image segmentation using iterative dilation scheme,
AUPIGITER. R. also discussed (16) segmentation using iterative watershed algorithm in $3 \mathrm{D}$ environment, which is suitable for medical image processing. ZHANG XIAO JING \& others discussed (17) segmentation using watershed algorithm, to be applied in medical area, using iterative erosion technique.

ZHUANG, H; \& others - (18) discussed methodology for smoothing (for the treatment of impulse Gaussian noises) using iterative close - open technique. SKOLNICK, M.M etc (19) discussed determination of centroids using iterative morphology.

J.G.POSTAIRE \& others (20) discussed the role of iterative morphology in cluster analysis. There are so many applications, where iterative morphology may be applied.

\section{MULTI SCALE SOFT MORPHOLOGY}

\subsection{Discussion On Multi Scale Soft Morphology}

In the process of understanding the objective world, the appearance of an object does not depend only on the object itself, but also on the scale that the observer used. It seems that appearance under a specific scale does not give sufficient information about the essence of the percept, we want to understand. If we use a different scale, to examine this percept, it will usually have a different appearance. So, this series of images and its changing pattern over scales reflect the nature of the percept.

The S.E. dimension can be anything. It depends upon situation, requirement, and context etc. It can be $1 / 1,2 / 2,3 / 3,4 / 4,5 / 5,6 / 6,7 / 7, \ldots \ldots \ldots$

In some situations, particularly square grid is chosen, it can be $3 / 3,5 / 5,7 / 7,9 / 9,11 / 11,13 / 13, \ldots \ldots$.

For example, it can be

$$
\left(\begin{array}{lll}
1 & 1 & 1 \\
1 & 1 & 1 \\
1 & 1 & 1
\end{array}\right)
$$

OR

$\left(\begin{array}{lllll}1 & 1 & 1 & 1 & 1 \\ 1 & 1 & 1 & 1 & 1 \\ 1 & 1 & 1 & 1 & 1 \\ 1 & 1 & 1 & 1 & 1 \\ 1 & 1 & 1 & 1 & 1\end{array}\right)$

OR

$$
\left(\begin{array}{lllllll}
1 & 1 & 1 & 1 & 1 & 1 & 1 \\
1 & 1 & 1 & 1 & 1 & 1 & 1 \\
1 & 1 & 1 & 1 & 1 & 1 & 1 \\
1 & 1 & 1 & 1 & 1 & 1 & 1 \\
1 & 1 & 1 & 1 & 1 & 1 & 1 \\
1 & 1 & 1 & 1 & 1 & 1 & 1 \\
1 & 1 & 1 & 1 & 1 & 1 & 1
\end{array}\right)
$$


The S.E.'s, having series, and in increasing size [like mentioned above] is called multi scale S.E.'s and the morphological approach (operations) dealing with multi scale S.E.'s is called multi scale morphology. As the size of the S.E. is more, its impact upon image will be more. For example, amount of expansion by applying dilation operation is more on an image, if we apply $\mathbf{5} / \mathbf{5}$ S.E., compared to amount of expansion of image, by dilating by $3 / 3$ S.E.

If Multi Scale Soft Morphology and iterative soft morphology are combined, a new area Multi Scale iterative Soft Morphology Emerges. [MSISM]

\subsection{Review On Multi Scale Soft Morphology}

Till now, some amount of research is done in this area, and it is applied in so many areas. In mathematical morphology also, a new area multi scale mathematical morphology is developed, and applied in so many areas like smoothening, edge enhancement, analysis of radar imagery, remote sensing, medical image processing etc.

PETROS MARAGOS entered into multi scale morphology, in addition to other areas. He explained about changes of shapes, as the scale is changed. He explained the applications of MSMM, and back ground mathematics. He explained about application of MSMM in skeletenization also. He extended these concepts to gray scale, also (21). MING - HUA CHEN \& PING - GAN YAN explained (22) Erosion, Dilation, Open, Close in multi scale environment, with diagrams (results), mathematical analysis, as well as symbolic conventions.

PAUL. T. JACKWAY etc. (49) provided one type of analysis in MSMM. They discussed how to relate the results of one scale with the results at different scale. They have provided this analysis with good examples, using Erosion/Dilation morphological operations. This paper discussed the B.G. theory, in one angle, relating to MSMM.

KUN WANG etc. proposed an algorithm, for edge detection in the presence of Gaussian noise \& salt - pepper noise in multi scale morphological environment. The experimental results are better than that of conventional algorithms (23). The same authors KUNWANG etc. proposed another algorithm for edge detection (24) which will function better in Gaussian, salt - paper noise environment, in MS morphological approach.

KIM WANG and others discussed an edge detection algorithm, in multi scale environment, which is suitable to apply on brain MRI, in noisy environment. (25).

Runway detector plays a very important role in synthetic vision system, which is helpful for pilots. But the infrared image constructed in this situation which will help pilot, will have heavy noise and bad contrast. Suitable to this situation, a multi scale morphological edge detector is proposed, which will help the pilot, for his, environmental awareness. So, MSMM is having, its applications, in aviation areas also. YANG SHANMIN and others discussed above MSMM concept. (26).

GAO LI etc (27) proposed an adaptive algorithm for edge detection of a color image (In HIS space) in MSMM environment. CHEN JIN LONG, etc. proposed another methodology for edge detection in multi structure and multi scale mathematical morphology environment (28).

ZHANG ANU \& others have proposed another algorithm (29) for identification of weak edges in Oct images using MSMM environment, XINGHUI ZAANG etc. (30) proposed another algorithm for edge detection in color image environment, using MSMM.

Another algorithm for smoothening is discussed using MSMM. (31). XU, YANLEI; ZHAO, JIYIN discussed (32) another algorithm, for edge detection, using MSMM, in noisy environment.

JIAN-HUI TAN etc. proposed (33) a new type of process using MSMM for smoothening of infrared imagery. It will have complexity due to noise. Using this methodology, they protected details also.

These MSMM techniques are extended to segmentation also. DEBAY LE, J. etc (34) extended MSMM for segmentation using adaptive technique and MARC DROSKE etc. also (35) used MSMM for segmentation. (36) $\mathrm{H}$ UANG, R. etc. discussed extension of MSMM to 3D. They discussed and designed algorithm for volume segmentation. For this purpose, they have designed spherical S. E.'s at various sizes. LETITIA, S; etc. applied MSMM for road segmentation from satellite aerial images (37).

JIANN-JONE CHEN etc. extended the MSMM to 3D segmentation, using dual (MS morphological) concepts (38).

SHU LI; etc. (39) designed water sheds segmentation algorithm, using MSMM, and applied to cell image segmentation, and got quality results. XU YING SHA; etc (40) proposed another water shed algorithm for segmentation of remote sensing images, in MSMM environment. It shows good results, by avoiding, over segmentation.

SHUWEI LI etc. (41) proposed method, to generate DTM and to maintain the terrain details, based on MSMM [here DTM means, Digital Terrain Model].

MSMM is having, application in medical area also. (42) DA WEI QI etc. shown an application in medical I.P. for edge detection in noisy environment, which gives better results, compared to traditional pictures. FEI ZHANG etc., given another algorithm (43), suitable for ECG analysis, in 
impulse noise environment using MSMM. DAWEI QI (44) proposed another algorithm, for medical analysis environment. JI - LE HU; etc. (45) proposed another algorithm, in ECG analysis, which provided suitable and good decisions, at critical points. It is a decision making algorithm regarding heart using MSMM.

Some relevant concepts are discussed in 46,47,48 which are related to soft mathematical morphology.

\section{5: EQUALITY IN BETWEEN ITERATIVE SOFT EROSION, ITERATIVE SOFT OPEN IN MULTI \\ SCALE ENVIRONMENT}

In this paper these equalities are discussed structuring element dimension wise. In this paper some important symbolic representation (or nomenclature) is also introduced and discussed.

In this context, referring paper 47 will be more helpful.

\section{1. $3 / 3$ Structuring Element}

\section{1.1 Threshold=1:}

Threshold value fixed at 1 .

$\left.\begin{array}{c}\text { Formula for iterative soft erosion } \\ \text { applied twice on an image }\end{array}\right\}=(E(1) E(1))$

$=(E(1) D(9))[\because E(1)=D(9)]$

$=O(1,9) \therefore(E(1))^{2}=O(1,9)$

Formula for iterative soft erosion)

applied four times on an image $\}$

$$
=(E(1) E(1) E(1) E(1))
$$

$=(E(1) D(9) E(1) D(9))[\because E(1)=D(9)]$

$=(O(1,9) O(1,9))$

$=(O(1,9))^{2} \therefore(E(1))^{4}=(O(1,9))^{2}$

Formula for iterative soft erosion)

applied six times on an image $\}$

$$
=(E(1) E(1) E(1) E(1) E(1) E(1))
$$

$=(E(1) D(9) E(1) D(9) E(1) D(9))[\because E(1)=D(9)]$

$=(O(1,9) O(1,9) O(1,9))$

$=(O(1,9))^{3} \therefore(E(1))^{6}=(O(1,9))^{3}$

Formula for

iterative soft erosion

applied 10 times on an image

$=(E(1) E(1) E(1) E(1) E(1) E(1) E(1) E(1) E(1) E(1))$

$=(E(1) D(9) E(1) D(9) E(1) D(9) E(1) D(9) E(1) D(9))$

$$
[\because E(1)=D(9)]
$$

$=(O(1,9) O(1,9) O(1,9) O(1,9) O(1,9))$

$=(O(1,9))^{5}$

\section{Formula for}

$$
\therefore(E(1))^{10}=(O(1,9))^{5}
$$

$$
\begin{aligned}
& \text { iterative soft erosion } \\
& \left.\begin{array}{c}
\text { applied } 2 n \text { times on } \\
\text { an image }
\end{array}\right\} \\
& =(E(1) E(1) E(1) E(1) E(1) E(1) \ldots \ldots E(1) E(1)) \\
& =\left(\begin{array}{ccccc}
1 & 2 & 3 & \ldots \ldots & n \\
& (1) D(9) E(1) D(9) E(1) D(9) & \ldots \ldots & E(1) D(9))
\end{array}\right. \\
& { }^{1}[\because E(1) \stackrel{2}{=} D(9)] 3 \quad \cdots \cdots \quad n
\end{aligned}
$$

$$
\begin{aligned}
& =(O(1,9) O(1,9) O(1,9) \ldots \ldots O(1,9)) \\
& =(O(1,9))^{n} \therefore(E(1))^{2 n}=(O(1,9))^{n}
\end{aligned}
$$

\section{1.2 Threshold=7:}

Threshold value fixed at 7 .

$\left.\begin{array}{c}\text { Formula for iterative soft erosion } \\ \text { applied twice on an image }\end{array}\right\}=(E(7) E(7))$

$=(E(7) D(3))[\because E(7)=D(3)]$

$=O(7,3) \therefore(E(7))^{2}=O(7,3)$

Formula for iterative soft erosion

applied four times on an image

$$
=(E(7) E(7) E(7) E(7))
$$

$=(E(7) D(3) E(7) D(3))[\because E(7)=D(3)]$

$=(O(7,3) O(7,3))$

$=(O(7,3))^{2} \therefore(E(7))^{4}=(O(7,3))^{2}$

\section{Formula for}

iterative soft erosion

applied six times on an image

$$
=(E(7) E(7) E(7) E(7) E(7) E(7))
$$

$=(E(7) D(3) E(7) D(3) E(7) D(3))[\because E(7)=D(3)]$

$=(O(7,3) O(7,3) O(7,3))$

$=(O(7,3))^{3} \therefore(E(7))^{6}=(O(7,3))^{3}$

Formula for

iterative soft erosion

$\left.\begin{array}{c}\text { applied ten times on } \\ \text { an image }\end{array}\right\}$

$=(E(7) E(7) E(7) E(7) E(7) E(7) E(7) E(7) E(7) E(7))$

$=(E(7) D(3) E(7) D(3) E(7) D(3) E(7) D(3) E(7) D(3))$

$$
[\because E(7)=D(3)]
$$

$=(O(7,3) O(7,3) O(7,3) O(7,3) O(7,3))$

$=(O(7,3))^{5} \therefore(E(7))^{10}=(O(7,3))^{5}$

Formula for

iterative soft erosion

$\left.\begin{array}{c}\text { applied } 2 n \text { times on } \\ \text { an image }\end{array}\right\}$

$=(E(7) E(7) E(7) E(7) E(7) E(7) \ldots \ldots E(7) E(7))$

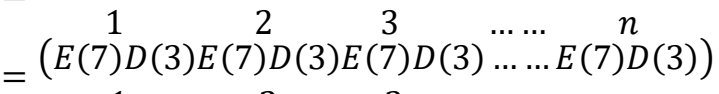

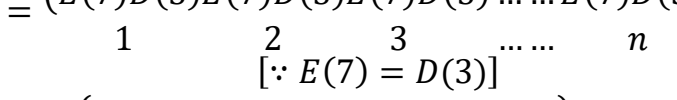

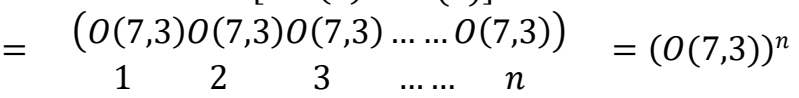

$$
\begin{aligned}
& \therefore(E(7))^{2 n}=(O(7,3))^{n}
\end{aligned}
$$

\section{1.3 General case:}

In general let threshold value $=m$

Formula for

iterative soft erosion

applied $2 n$ times on
an image

$=(E(m) E(m) E(m) E(m) E(m) E(m) \ldots \ldots E(m) E(m))$

$$
\begin{gathered}
110 \\
=E(m) D(10-m) E(m) D(10-m) E(m) D(10 \\
-m) E(m) D(10-m) \ldots \ldots
\end{gathered}
$$


$=O(m, 10-m) O(m, 10-m) O(m, 10-m) O(m, 10$

$=(O(m, 10-m))^{n}$

$$
-m) \ldots \ldots O(m, 10-m)
$$

$\therefore(E(m))^{2 n}=(O(m, 10-m))^{n}$

\section{$5.25 / 5$ Structuring Element:}

\section{2.1 Threshold=1:}

Threshold value fixed at 1 .

$\left.\begin{array}{c}\text { Formula for iterative soft erosion } \\ \text { applied twice on an image }\end{array}\right\}=(E(1) E(1))$

$=(E(1) D(25))[\because E(1)=D(25)]$

$=O(1,25) \therefore(E(1))^{2}=O(1,25)$

Formula for iterative soft erosion)

applied four times on an image $\}$

$$
=(E(1) E(1) E(1) E(1))
$$

$=(E(1) D(25) E(1) D(25))[\because E(1)=D(25)]$

$=(O(1,25) O(1,25))=(O(1,25))^{2}$ $\therefore(E(1))^{4}=$

$(O(1,25))^{2}$

Formula for iterative soft erosion) applied six times on an image $=(E(1) E(1) E(1) E(1) E(1) E(1))$

$=(E(1) D(25) E(1) D(25) E(1) D(25)) \quad[\because E(1)=$ $D(25)$

$=(O(1,25) O(1,25) O(1,25))=(O(1,25))^{3} \quad \therefore(E(1))^{6}=$ $(O(1,25))^{3}$

Formula for

iterative soft erosion

applied ten times on
an image

$=(E(1) E(1) E(1) E(1) E(1) E(1) E(1) E(1) E(1) E(1))$

$=$

$(E(1) D(25) E(1) D(25) E(1) D(25) E(1) D(25) E(1) D(25))$

$[\because E(1)=D(25)]$

$=(O(1,25) O(1,25) O(1,25) O(1,25) O(1,25))$

$=(O(1,25))^{5} \therefore(E(1))^{10}=(O(1,25))^{5}$

Formula for

iterative soft erosion

applied $2 n$ times on an image

$=(E(1) E(1) E(1) E(1) E(1) E(1) \ldots \ldots E(1) E(1))$

$=\left(\begin{array}{ccccc}1 & 2 & 3 & \ldots \ldots & n \\ E(1) D(25) E(1) D(25) E(1) D(25) & \ldots \ldots E(1) D(25))\end{array}\right.$

$\begin{array}{lllll}1 & 2 & 3 & \ldots \ldots & n\end{array}$

$[\because E(1)=D(25)]$

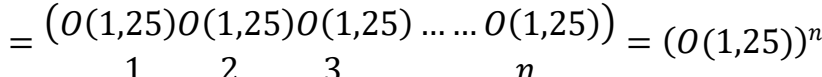

$$
\therefore(E(1))^{2 n}=(O(1,25))^{n}{ }^{n}
$$

\section{2.2 Threshold = 15:}

Threshold value fixed at 15 .

$\left.\begin{array}{c}\text { Formula for iterative soft erosion } \\ \text { applied twice on an image }\end{array}\right\}=(E(15) E(15))$

$=(E(15) D(11))[\because E(15)=D(11)]$

$=O(15,11) \therefore(E(15))^{2}=O(15,11)$

Formula for iterative soft erosion)

applied four times on an image

$$
=(E(15) E(15) E(15) E(15))
$$

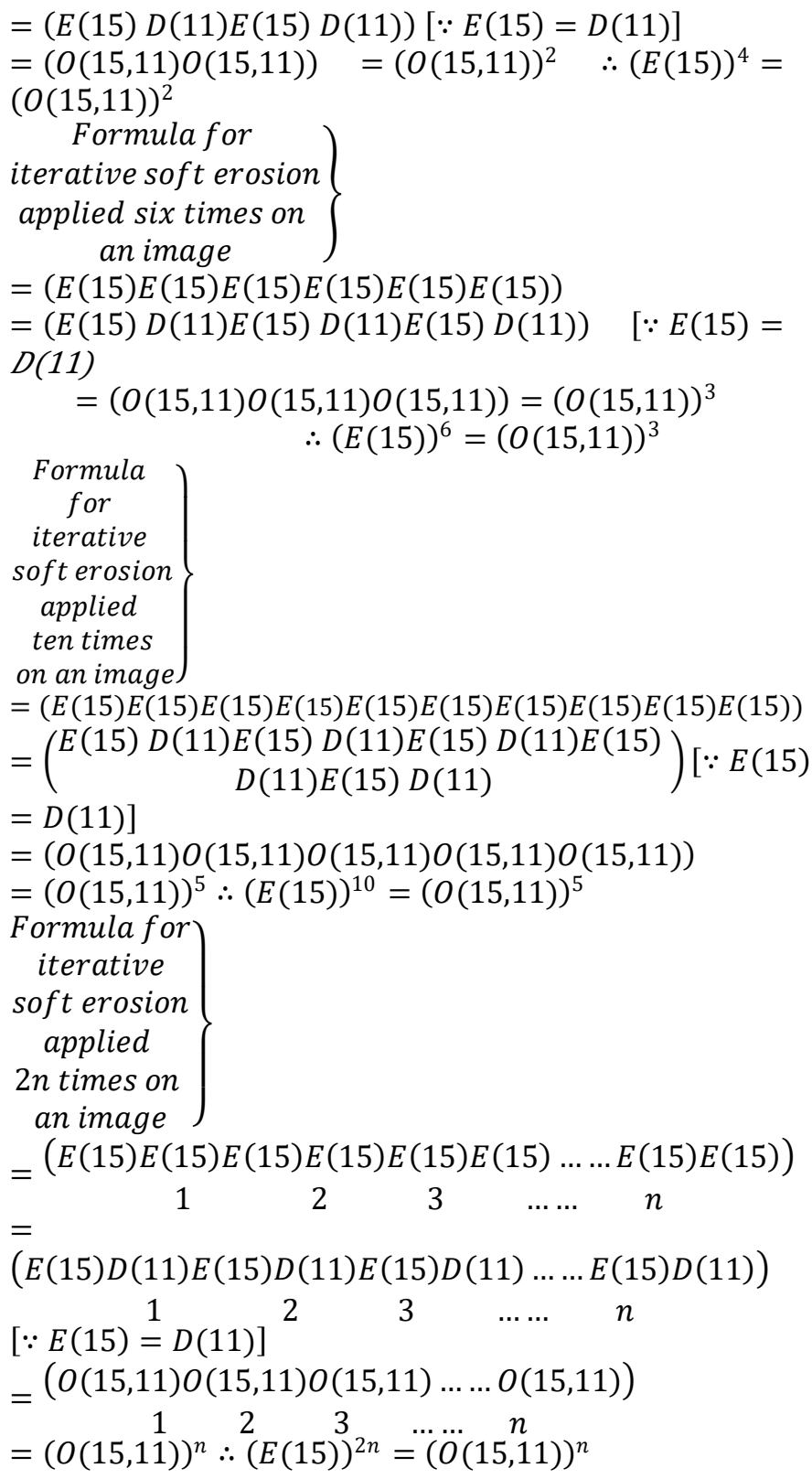

5. 2.3 General case:

$$
\begin{aligned}
& \text { In general let threshold value }=m \\
& \text { Formula for } \\
& \text { iterative soft erosion } \\
& \text { applied } \\
& 2 n \text { times on } \\
& \text { an image } \\
& =(E(m) E(m) E(m) E(m) E(m) E(m) \ldots \ldots E(m) E(m)) \\
& \begin{array}{llllll}
1 & 2 & 3 & \ldots & n
\end{array} \\
& =E(m) D(26-m) E(m) D(26-m) E(m) D(26 \\
& -m) E(m) D(26-m) \ldots \ldots
\end{aligned}
$$




\section{$5.37 / 7$ Structuring Element:}

\subsubsection{Threshold $=1$ :}

Threshold value fixed at 1 .

$\left.\begin{array}{c}\text { Formula for iterative soft erosion } \\ \text { applied twice on an image }\end{array}\right\}=(E(1) E(1))$

$=(E(1) D(49))[\because E(1)=D(49)]$

$=O(1,49) \therefore(E(1))^{2}=O(1,49)$

Formula for iterative soft erosion)

applied four times on an image

$$
=(E(1) E(1) E(1) E(1))
$$

$=(E(1) D(49) E(1) D(49))[\because E(1)=D(49)]$

$=(O(1,49) O(1,49))$

$=(O(1,49))^{2} \therefore(E(1))^{4}=(O(1,49))^{2}$

Formula for iterative soft erosion)

applied six times on an image $\}$

$$
=(E(1) E(1) E(1) E(1) E(1) E(1))
$$

$=(E(1) D(49) E(1) D(49) E(1) D(49)) \quad[\because E(1)=$ $D(49)$

$=(O(1,49) O(1,49) O(1,49))=(O(1,49))^{3} \quad \therefore(E(1))^{6}=$

$(O(1,49))^{3}$

Formula for

iterative soft erosion applied

ten times on an image

$=(E(1) E(1) E(1) E(1) E(1) E(1) E(1) E(1) E(1) E(1))$

$=$

(E(1) $D(49) E(1) D(49) E(1) D(49) E(1) D(49) E(1) D(49))$

$[\because E(1)=D(49)]$

$=(O(1,49) O(1,49) O(1,49) O(1,49) O(1,49))$

$=(O(1,49))^{5} \therefore(E(1))^{10}=(O(1,49))^{5}$

Formula for

iterative soft erosion

applied $2 n$ times on an image

$=(E(1) E(1) E(1) E(1) E(1) E(1) \ldots \ldots E(1) E(1))$

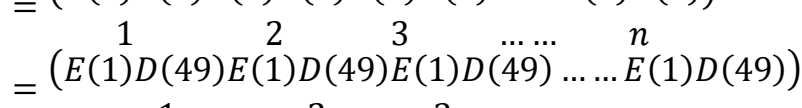

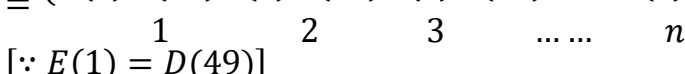

$=(O(1,49) O(1,49) O(1,49) \ldots \ldots O(1,49))$

$=(O(1,49))^{n} \quad \therefore(E(1))^{2 n}=(O(1,49))^{n}$

\subsection{Threshold $=35$ :}

Threshold value fixed at 35 .

Formula for iterative soft erosion applied twice on an image $\}=(E(35) E(35))$

$=(E(35) D(15))[\because E(35)=D(15)]$

$=O(35,15) \therefore(E(35))^{2}=O(35,15)$

Formula for iterative soft erosion)

applied four times on an image $=(E(35) E(35) E(35) E(35))$

$=(E(35) D(15) E(35) D(15))[\because E(35)=D(15)]$

$=(O(35,15) O(35,15))=(O(35,15))^{2} \quad \therefore(E(35))^{4}=$ $(O(35,15))^{2}$
Formula for

$\left.\begin{array}{c}\text { iterative soft erosion } \\ \text { applied six times on } \\ \text { an image }\end{array}\right\}$

$=(E(35) E(35) E(35) E(35) E(35) E(35))$

$=(E(35) D(15) E(35) D(15) E(35) D(15)) \quad[\because E(35)=$

$D(15)]$

$=(O(35,15) O(35,15) O(35,15))=(O(35,15))^{3}$

Formula for $\therefore(E(35))^{6}=(O(35,15))^{3}$

iterative

soft erosion

applied

ten times on

an image

$=(E(35) E(35) E(35) E(35) E(35) E(35) E(35) E(35) E(35) E(35))$

$=$

$(E(35) D(15) E(35) D(15) E(35) D(15) E(35) D(15) E(35) D(15))$

$[\because E(35)=D(15)]$

$=(O(35,15) O(35,15) O(35,15) O(35,15) O(35,15))$

$=(O(35,15))^{5} \therefore(E(35))^{10}=(O(35,15))^{5}$

Formula for

iterative

soft erosion

applied

$2 n$ times on

an image

$=(E(35) E(35) E(35) E(35) E(35) E(35) \ldots \ldots E(35) E(35))$

$\begin{array}{llllll}1 & 2 & 3 & \ldots \ldots & n\end{array}$

$=$

$(E(35) D(15) E(35) D(15) E(35) D(15) \ldots \ldots E(35) D(15))$

$[\because E(35) \stackrel{1}{=} \quad 2 \quad 3(15)]^{2} \quad \ldots \ldots . \quad n$

$=(O(35,15) O(35,15) O(35,15) \ldots \ldots O(35,15))$

$=(O(35,15))^{n} \quad \therefore(E(35))^{2 n}=(O(35,15))^{n}$

\subsubsection{General case:}

In general let threshold value $=m$

Formula for

iterative soft erosion applied

$2 n$ times on an image

$=(E(m) E(m) E(m) E(m) E(m) E(m) \ldots \ldots E(m) E(m))$

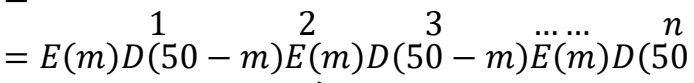

$-m) E(m) D(50-m) \ldots \ldots$ $E(m) D(50-m)$

$=O(m, 50-m) O(m, 50-m) O(m, 50-m) O(m, 50$

$=(O(m, 50-m))^{n}$ $-m) \ldots \ldots O(m, 50-m)$

So $(E(m))^{2 n}=(O(m, 50-m))^{n}$

\section{$5 . .49$ Structuring Element:}

\subsubsection{Threshold=1:}

Threshold value fixed at 1 . 
Formula for iterative soft erosion applied twice on an image $\}=(E(1) E(1))$

$=(E(1) D(81))[\because E(1)=D(81)]$

$=O(1,81) \therefore(E(1))^{2}=O(1,81)$

Formula for iterative soft erosion)

applied four times on an image $\}$ $=(E(1) E(1) E(1) E(1))$

$=(E(1) D(81) E(1) D(81))[\because E(1)=D(81)]$

$=(O(1,81) O(1,81))$

$=(O(1,81))^{2} \therefore(E(1))^{4}=(O(1,81))^{2}$

Formula for iterative soft erosion) applied six times on an image $\}$ $=(E(1) E(1) E(1) E(1) E(1) E(1))$

$=(E(1) D(81) E(1) D(81) E(1) D(81)) \quad[\because E(1)=$ $D(81)$

$=(O(1,81) O(1,81) O(1,81))$

$=(O(1,81))^{3} \therefore(E(1))^{6}=(O(1,81))^{3}$

Formula for

iterative

soft erosion applied

ten times on an image

$=(E(1) E(1) E(1) E(1) E(1) E(1) E(1) E(1) E(1) E(1))$

$=$

$(E(1) D(81) E(1) D(81) E(1) D(81) E(1) D(81) E(1) D(81))$

$[\because E(1)=D(81)]$

$=(O(1,81) O(1,81) O(1,81) O(1,81) O(1,81))$

$=(O(1,81))^{5} \therefore(E(1))^{10}=(O(1,81))^{5}$

Formula for iterative soft erosion) applied $2 n$ times on an image $\}$

$=(E(1) E(1) E(1) E(1) E(1) E(1) \ldots \ldots E(1) E(1))$

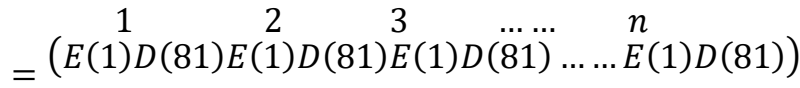

$[\because E(1)=D(81)] \quad \begin{array}{lllll}1 & 3 & \cdots \cdots & n\end{array}$

$=(O(1,81) O(1,81) O(1,81) \ldots \ldots O(1,81))$

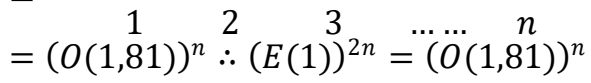

\section{4.2 Threshold $=60$ :}

Threshold value fixed at 60 .

$\left.\begin{array}{c}\text { Formula for iterative soft erosion } \\ \text { applied twice on an image }\end{array}\right\}=(E(60) E(60))$

$=(E(60) D(22))[\because E(60)=D(22)]$

$=O(60,22) \therefore(E(60))^{2}=O(60,22)$

Formula for iterative soft erosion)

applied four times on an image $=(E(60) E(60) E(60) E(60))$

$=(E(60) D(22) E(60) D(22))[\because E(60)=D(22)]$

$=(O(60,22) O(60,22))$

$=(O(60,22))^{2} \therefore(E(60))^{4}=(O(60,22))^{2}$

\section{Formula for}

iterative soft erosion

applied six times on an image

$=(E(60) E(60) E(60) E(60) E(60) E(60))$

$=(E(60) D(22) E(60) D(22) E(60) D(22)) \quad[\because E(60)=$

$D(22)$

$=(O(60,22) O(60,22) O(60,22))$

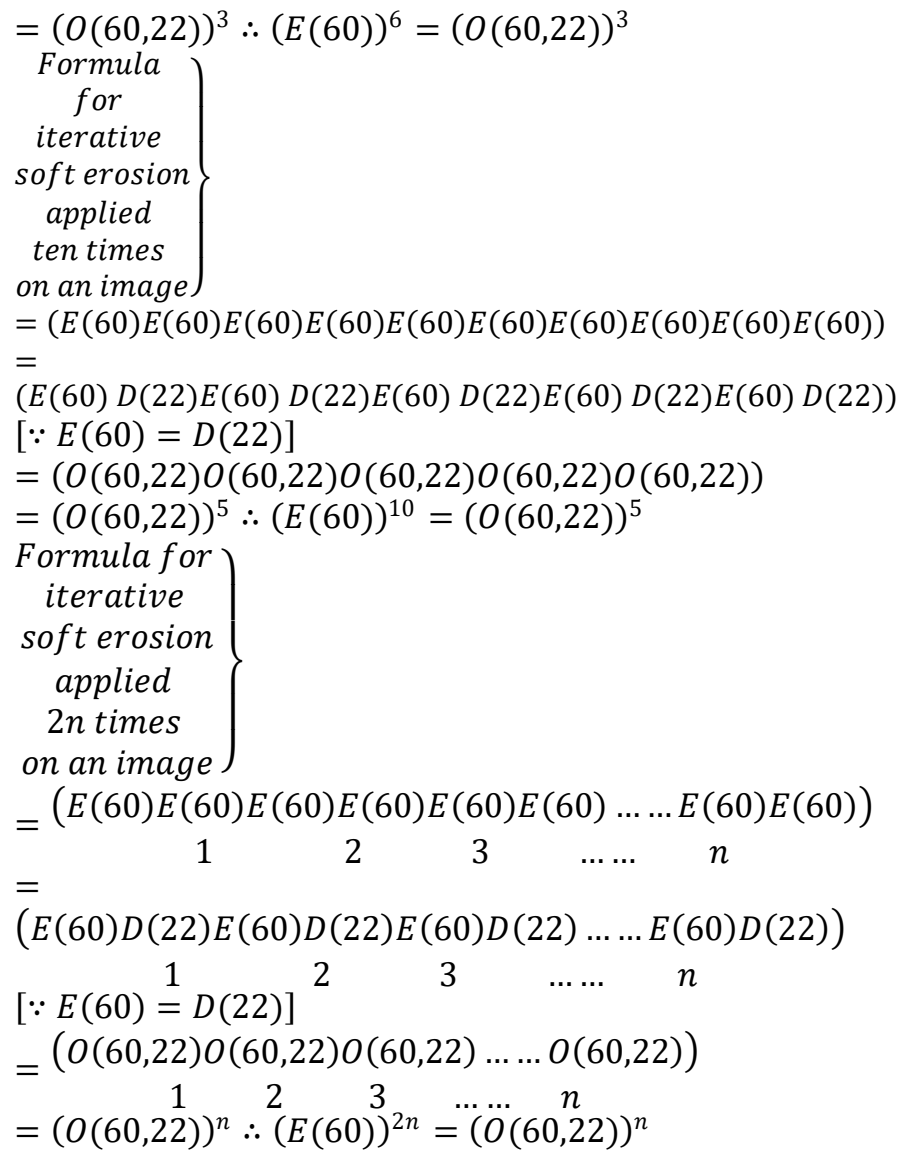

\section{4.3 General case:}

In general let threshold value $=m$

Formula for

iterative

soft erosion

applied

$2 n$ times on

an image

$=(E(m) E(m) E(m) E(m) E(m) E(m) \ldots \ldots E(m) E(m))$

$1 \quad \begin{gathered}2 \\ =E(m) D(82-m) E(m) D(82-m) \\ -m(m) D(82\end{gathered}$
$-m) E(m) D(82-m) \ldots \ldots$

$E(m) D(82-m)$

$=O(m, 82-m) O(m, 82-m) O(m, 82-m) O(m, 82$

$=(O(m, 82-m))^{n}$ $-m) \ldots \ldots O(m, 82-m)$

$\therefore(E(m))^{2 n}=(O(m, 82-m))^{n}$

In the same way the formulae may be developed for $11 / 11$,

$13 / 13,15 / 15,17 / 17 \ldots$. structuring elements.

5. 5 General case: $w / w$ structuring element size:

Formula for

iterative soft erosion applied

$2 n$ times on an image

$=(E(m) E(m) E(m) E(m) E(m) E(m) \ldots \ldots E(m) E(m))$ 


$$
\begin{aligned}
& =E(m) D\left(w^{2}+1-m\right) E(m) D\left(w^{2}+1\right. \\
& \quad-m) \ldots \ldots E(m) D\left(w^{2}+1-m\right) \\
& =O\left(m, w^{2}+1-m\right) O\left(m, w^{2}+1-m\right) \ldots \ldots O\left(m, w^{2}+1\right. \\
& \quad-m) \\
& =\left(O\left(m, w^{2}+1-m\right)\right)^{n} \\
& \quad>\quad \therefore(E(m))^{2 n}=\left(O\left(m, w^{2}+1-m\right)\right)^{n}
\end{aligned}
$$

\section{RESULTS AND DISCUSSIONS}

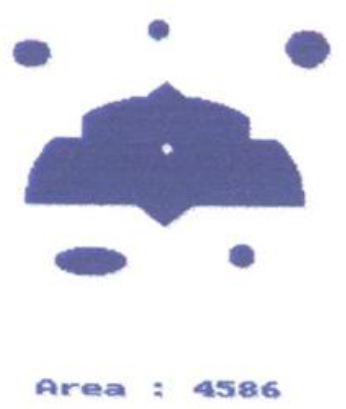

Semi-circle

In this section, the above discussed formulae are processed and results are shown and above formulae are verified practically.. They are verified by applying iterative soft morphological operations in multi scale environment on a few images. But in this paper a sample outputs only are presented, and equality is explained. These soft morphological operations are applied on the above semicircle, and results are tabulated in the following tables. The results of semi-circle are taken and there are tabulated in a systematic way. Initially the results are presented in $3 / 3$ environment, and equality in between iterative soft erosion and iterative soft open are established.

Here, the erosion twice with threshold value is equal to one, is equivalent to open at thresholds 1 and 9 . We get the same value, which is equal to 3469 , in $3 / 3$ window environment.

Here, the erosion four times with threshold value is equal to three, is equivalent to open twice at thresholds 3 and 7 . We get the same value, which is equal to 3199 , in $3 / 3$ window environment.

In the same way here, the erosion six times with threshold value is equal to four, is equivalent to open at thresholds 4 and 6 , three times. We get the same value, which is equal to 3846 , in $3 / 3$ window environment.

\begin{tabular}{|c|c|c|c|c|}
\hline \multicolumn{5}{|c|}{ Table relating iterative soft erosion and iterative soft open } \\
\hline \multicolumn{5}{|c|}{$3 / 3$ window environment } \\
\hline $\begin{array}{c}\text { Threshold value of } \\
\text { soft erosion. }\end{array}$ & $\begin{array}{c}\text { No of iterations of } \\
\text { soft erosions }\end{array}$ & $\begin{array}{l}\text { Threshold values of } \\
\text { equivalent Soft open. }\end{array}$ & $\begin{array}{l}\text { The no of iterations of } \\
\text { equivalent Soft open. }\end{array}$ & value \\
\hline 1 & 2 & 1,9 & 1 & 3469 \\
\hline 1 & 4 & 1,9 & 2 & 2588 \\
\hline 1 & 6 & 1,9 & 3 & 1896 \\
\hline 2 & 2 & 2,8 & 1 & 3723 \\
\hline 2 & 4 & 8,2 & 2 & 2979 \\
\hline 2 & 6 & 2,8 & 3 & 2357 \\
\hline 3 & 2 & 3,7 & 1 & 3850 \\
\hline 3 & 4 & 3,7 & 2 & 3199 \\
\hline 3 & 6 & 3,7 & 3 & 2687 \\
\hline 4 & 2 & 4,6 & 1 & 4353 \\
\hline 4 & 4 & 4,6 & 2 & 4096 \\
\hline 4 & 6 & 4,6 & 3 & 3846 \\
\hline 5 & 2 & 5,5 & 1 & 4583 \\
\hline 5 & 4 & 5,5 & 2 & 4583 \\
\hline 6 & 2 & 6,4 & 1 & 4704 \\
\hline 6 & 4 & 6,4 & 2 & 4780 \\
\hline 6 & 6 & 6,4 & 3 & 4860 \\
\hline 8 & 2 & 8,2 & 1 & 5526 \\
\hline 8 & 4 & 8,2 & 2 & 6540 \\
\hline 9 & 4 & 9,1 & 2 & 7330 \\
\hline 9 & 6 & 9,1 & 3 & 8982 \\
\hline 9 & 8 & 9,1 & 4 & 10807 \\
\hline
\end{tabular}

In the same way results of semi circle in $5 / 5$ environment is also tabulated.

The following tables provide information of iterative soft erosion and iterative soft open in $3 / 3$ environment and $5 / 5$ environment. The image taken is semi circle. 


\begin{tabular}{|c|c|c|c|c|}
\hline \multicolumn{5}{|c|}{ Table relating iterative soft erosion and iterative soft open } \\
\hline \multicolumn{5}{|c|}{$5 / 5$ window environment } \\
\hline $\begin{array}{c}\text { Threshold value of } \\
\text { soft erosion. }\end{array}$ & $\begin{array}{c}\text { No of iterations of } \\
\text { soft erosion s }\end{array}$ & $\begin{array}{c}\text { Threshold values of } \\
\text { equivalent Soft open. }\end{array}$ & $\begin{array}{c}\text { The no of iterations of } \\
\text { equivalent Soft open. }\end{array}$ & value \\
\hline 1 & 2 & 1,25 & 1 & 2588 \\
\hline 1 & 4 & 1,25 & 2 & 1269 \\
\hline 1 & 6 & 1,25 & 3 & 267 \\
\hline 1 & 8 & 1,25 & 4 & 0 \\
\hline 2 & 2 & 2,24 & 1 & 2759 \\
\hline 2 & 4 & 2,24 & 2 & 1498 \\
\hline 2 & 6 & 2,24 & 3 & 425 \\
\hline 2 & 8 & 2,24 & 4 & 4 \\
\hline 23 & 2 & 23,3 & 1 & 6709 \\
\hline 23 & 6 & 23,3 & 2 & 9290 \\
\hline 23 & 2 & 23,3 & 3 & 12151 \\
\hline 24 & 4 & 24,2 & 1 & 6978 \\
\hline 24 & 6 & 24,2 & 2 & 10020 \\
\hline 24 & 2 & 24,2 & 3 & 13347 \\
\hline 25 & 4 & 25,1 & 1 & 7330 \\
\hline 25 & 6 & 25,1 & 2 & 10807 \\
\hline 25 & & 25,1 & 3 & 14297 \\
\hline
\end{tabular}

\section{CONCLUSION}

In this paper a fundamental rule called EQUALITY is discussed in multi scale and iterative environment. It will fill up gap , on the fundamentals of mathematical soft morphology. Till now applications are discussed in various papers by various researchers, but fundamental properties are not touched. More over iterative morphology is having broad applications. so discussion of fundamental property in this context, will lead to development of this area. Understanding of fundamental properties of any area will lead to development and expansion of that area, which will lead to excellent applications.

\section{REFERENCES}

[1] J. Serra-Image Analysis and Mathematical Morphology.

[2] Matheron - Mathematical Morphology

[3] Robert. M. Haralick, Stanley R. Sternberg, Xinhua Zhuang (July 1987) Image Analysis using Mathematical Morphology, IEEE Transactions on PAMI, Vol. 9, No. 4.

[4] H. J. A. M. Heijmans and C. Ronse. (1990) The Algebraic Basis of Mathematical Morphology. 1. Dilations and Erosions, IEEE Transactions on Computer Vision, Graphics and Image Processing

[5] Michel A. Zmoda and Louis. A.Tamburino - Efficient algorithms for the soft morphological operators. --- IEEE Tr. of Pami - Vol. 18 - No.11 November 1996.

[6] A generalized approach for handling equality and duality properties in soft morphology...PhD thesis of KOMPELLA VENKATA RAMANA
[7] Shih, F. Y; Pu. C. C; ---Analysis of the properties of soft morphological filtering using threshold decomposition.-IEEE Tr. of S.P.Vol. 43 - No.2 November 1995.

[8] Pu. C. C; - Threshold decomposition algorithm for gray scale soft morphological operations --- II -Erosion;--- 5th International conference on Image processing and its applications - 1995 .

[9] Pauli Kuosmanen \& Jaakko Astola. - Soft morphological filtering --- Journal of mathematical imaging and vision 1995.

[11] Koivisto, P; Huttunen; H. Kuosmanen, P;--- Optimal soft morphological filtering under breakdown probability constraints --- IEEE Int. Sym. On C \& S - 1996.

[12] Lipeng Wang; Hanbin Wang; -- Implementation of a soft morphological filter based on GPU frame work - Int. Conf. on Bioinformatics and Biomedical Engineering 2011.

[13] Chanf - Chefchaouni, M; Schonfeld; D.-Convergence criteria for iterative non linear filters.- S. M. C - IEEE Int. Conf. - 1992.

[14] Jiwei Yuan; Zhongke Shi.- A new segmentation method for image sequence of traffic scenes.- WCICA- fifth world congress-2004.

[15] Yong Xia; Dagan Feng; Rongchun Zhao.Morphology- based multi fractal estimation for texture segmentation. IEEE Trans of Image Processing - Vol. 15. No - 3. -2006.

[16] Audigier, R.; Lotufo, R.; Falcao, A.;- On integrating iterative segmentation by watershed with tridimensional visualization of MRI's. - IEEE Conf. on CG \& IP- 2004. 
[17] ZHANG XIA O - JING; SUN WAN - RONG --- A new algorithm for watershed segmentation of cells in marrow - IEEE - INT - Conf - 2005

[18] ZHUANG, H; HAMANO, F - A new type of effective morphologic edge detectors. IEEE - Conf.-1988.

[19] SKOLNICK, M. M; BROWN, R. H.; BHAGVATI, C; WOLF, B. R; -- IEEE Conf. on C \& S - 1989

[20] J.G.POSTAIRE; R. D. ZHANG; C. LECOCQ BOTTE.- Cluster analysis by binary morphology. - Pami Vol. 15 - No.2. - Feb 1993.

[21] PETROS; MARAGOS; Pattern spectrum and multi scale shape representation.

- IEEE Trans. of Pami - Vol. 11. No.-7. July -1989

[22] MING - HUA CHEN \&PING - FAN.YAN. --- A Multi scaling approach based on morphological filtering. IEEE Trans. of Pami - Vol. 11. No-7. July -1989

[23] KUN WANG; JIANHUA WU; LIQUN GAO; ZHAOYU PIAN; LI GUO--- Magnetic resonance images, edge detection , based on multi - scale morphology. --INT. CONF. ON - COMPLEX MEDICAL ENGINEERING - CME - IEEE - 2007

[24] KUN WANG; LIQUN GAO; ZHENGANG SHI; LI GUO; ZHAOYA PIAN. - An edge detection algorithm based on "multi scale morphology" --- IEEE CONF. ON INDUSTERIAL ELECTRONICS \& APPLICATIONS. ICIEA - 2007

[25] Kim Wang; Jianhga Wu; Zhaoyu pian: --- Edge detection algorithm for magnetic resonance images based on multi - scale morphology. Int. Conf. on Control and Automation 2007.

[26] Yang Shanmin; Feng Ziliang; Zhang xiuqiong; Yang hongyu --- A multi - scale morphologic runway detector--2nd IEEE Int. Conf. on Computer Engineering and Technology --- ICCET - 2010.

[27] Gao Li; Ling Xiao-ming; --- Colour edge detection based on mathematical morphology in HSI Space. --- IEEE Int. Conf. on Computer and Information application --ICCIA - 2010.

[28] Chen Jin Long; Zhang Bin; Qi Yingjian; -- image edge detection method based on multi - structure and multi scale mathematical morphology. --- Int. conf. on multimedia technology - ICMT --- 2010.

[29] Zhang Ang; Huang SI Ziw Ei; Tian Xia Olin; Sun Yan --- Kui; --- Morphologic weak edge detection by Multi structure and Multi - Scale in Anterior Chamber OCT images.--- 4th Int. Cong. - IEEE - Image and signal processing ---CISP- 2011.

[30] Xingh uI Zhang; Jiuying Li.-A method of Colour edge detection using mathematical morphology .ICCSNT-IEEE Int. Conf.

[31] Morphology based symbolic image modeling, multi scale nonlinear smoothing, and pattern spectrum Computer Society Conference on computer vision and pattern recognition--- 1988.

[32] Xu, Yanlei; Zhao, Jiyin;--- Noisy image edge detection based on multi - scale and multi - structuring element order morphology transformation.--- IEEE Conference on image and signal processing---2008.
[33] Jian - Hui Tan; Bao - Chang Pan - A new algorithm for infrared image restoration based on multi - scale morphological wavelet and Hopfield neural network. IEEE International conference on Wavelet analysis and pattern recognition - 2010 .

[34] Debayle, J; Ecolenat; Pinoli, J - Multi - scale image filtering and segmentation by means of adoptive neighborhood mathematical morphology.- IEEE conference on image processing 2005.

[35] Marc droske; martin Rumpf;-- Multi - scale joint segmentation and registration of image morphology. IEEE Tr. of Pami - Vol. 29-No. 12 December 2007.

[36] Huang, R; Lum, E; MA, K.L; -- Multi - Scale morphological volume segmentation and Visualization. 6th Asia - Pacific Symposium on Visualization - 2007.

[37] Letitia, S; Monie, E.C; - Road segmentation from satellite aerial images by means of adaptive neighborhood mathematical morphology.--Int. Conf. on Computer and Communication Engineering 2008.

[38] Jia nn - Jone chen; Chun - rongsu. - Volume image segmentation by dual multi - Scale morphological reconstructions.--- 5th IEEE Int. conf. on IIH-MSP-2009.

[39]Shu Li; Lei Wu; Yang Sun. - Cell image Segmentation based on an improved watershed transformation. - IEEE Int. Conf on computational Aspects of Social Networks - 2010.

[40] Xu Yingsha; Fang Zhaolin. - Improved Segmentation of Remote Sensing Images Based on WaterShed Algorithm - IEEE International Conference on Consumer Electronics, Communications and networks. - 2011

[41] Shuwei Li; Lei Yan; Huabo sun. - A Filtering Method for Generating DTM based on Multi - Scale Mathematical Morphology. - Int. Conf. on Mechatronics and Automation. $-2011$.

[42] Divyendu sinha; Charles R. Giardina - Discrete black and white object recognition via morphological functions --IEEE Tr. of Pami - Vol. 12 - No.3 March 1990.

[43]Xia oli wane and Gilles Bertrand - Some sequential algorithms for a generalized distance transformation based on MINKOWSKI operations. - IEEE Tr. of Pami - Vol. 14 No.11 November 1992.

[44] Kyeong - Ryeol - park; Chung - Nimlee; --- Scale Space using mathematical morphology.--- IEEE Tr. of Pami - Vol. 18 No.11 November 1996.

[45] Lei Chen; Xian - Wu Hyang; Xing - Rong Zhong; --Morphological algorithms for face detection. - IEEE Int. Conf. on VLSI design and Video technology. - 2005.

[46] KOMPELLA VENKATA RAMANA...... Duality in soft dilation in Multi Scale Soft Morphological Environment......INTERNATIONAL ADVANCED RESEARCH JOURNAL IN SCIENCE, ENGINEERING AND TECHNOLOGY.... Vol 3,Issue 2,Feb 16

[47] KOMPELLA VENKATA RAMANA......Equality in Soft Erosion and Soft Dilation in Multi Scale Environment.... IJARCCE..... Vol 5 ,Issue 2, Feb 16.

[48] KOMPELLA VENKATA RAMANA.......DUALITY IN SOFT EROSION IN MULTI SCALE SOFT MORPHOLOGICAL ENVIRONMENT .......IJARCSSE..... vol 6, issue 2, FEB 16 


\section{BIOGRAPHY}

The author Kompella Venkata Ramana has done his B.E (E.C.E) and M.E (COMPUTER ENGINEERING) and Ph.D from ANDHRA UNIVERSITY, VISAKHAPATNAM, INDIA. He has started his carrier as LECTURER in N.I.T. (W). Later he shifted to ANDHRA UNIVERSITY. At present he is working as ASSOCIATE PROFESSOR in the department of computer science \&systems engineering in ANDHRA UNIVERSITY. His areas of interest are Image Processing, Formal languages and Automata theory, compiler design and Systems Programming. He has written books on the above areas. He has experience of more than twenty five years in teaching and guided more than one hundred Thesis in M.Tech. Level,majority of them are in image processing.

He has done his $\mathrm{Ph} . \mathrm{D}$ in computer engineering (image processing...mathematical soft morphology.) 